Recepción: 27/06/2014

Evaluación: 01/09/2014

Aprobación: 04/11/2014

Artículo de Reflexión

\title{
LA FUNDACIÓN URDIMBRE DE LA CIUDAD DE SAN JUAN DE PASTO, A LA LUZ DE LA PEDAGOGÍA SOCIAL
}

\author{
Bárbara 0jeda Cortés* \\ Universidad Mariana \\ Linea de Investigación: Historia de la Educación superior latinoamericana \\ barbara7980@gmail.com
}

\begin{abstract}
RESUMEN
Este artículo es resultado de la tesis doctoral Presencia de la pedagogía social en los programas socio educativos de la obra social El Carmen y Fundación Urdimbre del municipio de Pasto, cuyo proceso de revisión bibliográfica, selección de información y análisis, se sustentó en el paradigma cualitativo, en el método de teoría fundamentada de Strauss y Corbin y en el empleo de la técnica de análisis de contenido; con la intención de dar respuesta a la categoría específica: componente pedagógico de las dos fundaciones, la cual contiene una sub categoría denominada "perfil, características, competencias u otros del personal que educa a la población atendida".

Desde estas claridades y para la elaboración de este texto, se tomó como referencia la Fundación URDIMBRE, por los elementos que evidenció en el momento de realizar procesos de comparación, acercamiento y distanciamiento de los perfiles, características y competencias de las y los educadores sociales, propósitos y dinámicas formativas que sustenta la pedagogía social para la educación y cualificación docente, en procesos socio-educativos.
\end{abstract}

A raíz de este proceso, se puede sustentar, que la Ciudad de San Juan de Pasto cuenta con la Fundación URDIMBRE que, por sus características y

\footnotetext{
* Doctora en Ciencias de la Educación, Universidad de Nariño/RUDECOLOMBIA (Novena Cohorte, 2014), Especialista en Docencia Universitaria, Universidad de Nariño; Licenciada en Ciencias Sociales, Universidad de Nariño; miembro del Grupo de Investigación para el Desarrollo de la Educación y Pedagogía (GIDEP), Facultad de Educación, y del Grupo de Investigación Multiverso, (Facultad de Ciencias Humanas, Universidad de Nariño), docente de la Universidad Mariana, Facultad de Educación, Programa de Ciencias Sociales y Maestría en Educación de la Universidad de Nariño, correo electrónico: barbara7980@gmail.com
} 
particularidades socio-educativas, puede situarse de manera práctica, mas no teórica, en la Educación Social, puesto que el proceso de pesquisa y reflexión reveló que la entidad adelanta procesos de cualificación docente e investigación social relacionados con los principios y finalidades de la Pedagogía Social, destinados a la optimización de la atención y educación de diferentes grupos sociales que se encuentran en situación de vulnerabilidad, desplazamiento y en calle, al responder a sus necesidades, tiempos, intereses y expectativas, desde acciones y metodologías que posibilitan la formación integral, atención psicosocial familiar y trabajo en diferentes escenarios para la integración de los actores que hacen parte del mundo de las niños, niños y jóvenes que asisten a la fundación.

Palabras claves: Fundación URDIMBRE, perfil, competencias y cualificación de educadores, Pedagogía Social.

\title{
THE “URDIMBRE” FOUNDATION OF THE CITY OF SAN JUAN OF PASTO IN THE LIGHT OF SOCIAL PEDAGOGY
}

\begin{abstract}
This article is the result of the doctoral thesis "PRESENCE OF SOCIAL PEDAGOGY IN THE "URDIMBRE" FOUNDATION and CARMEN SOCIAL WORK EDUCATIONAL PROGRAMS OF THE MUNICIPALITY OF PASTO" whose process of literature review, selection of information and analysis was defended on the basis of a qualitative paradigm grounded in the theory method of Strauss and Corbin, and in the use of the technique of content analysis, with the intention of responding to the specific category: the educational component of the two foundations, which contains a sub category called "profile, characteristics, competencies or other features of the staff that educates the population served."

In order to clarify and for the preparation of this text, reference was made to the "URDIMBRE" Foundation for the elements evident at the time of comparison processes, zooms in on profiles, characteristics and competencies of the social educators, purposes and dynamic training that support social pedagogy for teacher education and qualification in social and educational processes.
\end{abstract}

Following this process, it can be stated that the City of "San Juan de Pasto", possesses the "URDIMBRE" Foundation, whose characteristics and social and educational characteristics are situated from a practical rather than a theoretical perspective in Social Education, since the process of research and reflection revealed that the aforementioned entity advances teaching qualification processes, and social research related to the principles and purposes of the Social Pedagogy aimed at optimizing the care and education of different social groups in situations of vulnerability, displacement in the street; responding to their needs, 
times, interests and expectations from actions and methodologies that enable comprehensive training, psychosocial family care, and work in different scenarios for the integration of the actors who are part of the world of children and young people attending the foundation.

Keywords: "URDIMBRE" Foundation Profile, skills and qualifications of educators, Social Pedagogy.

\title{
A FUNDAÇÃO URDIMBRE DA CIDADE DE SAN JUAN DE PASTO, NA LUZ DA PEDAGOGIA SOCIAL
}

\begin{abstract}
RESUMO
0 artigo é resultado da tese de doutorado "PRESENÇA DA PEDAGÓGIA SOCIAL NOS PROGRAMAS SÓCIO EDUCATIVOS DA OBRA SOCIAL EL CARMEN E FUNDAÇÃO URDIMBRE DA CIDADE DE PASTO" cujo processo de revisão bibliográfica, seleção de informação e análise da mesma, se apoiou no paradigma qualitativo, no método de teoria fundamentada de Strauss e Corbin, e na utilização da técnica de análise de conteúdo; com a intenção de responder a categoría especificada: componente pedagógico das duas fundações, que contém uma sub categoría chamada "perfil, características, competências ou outros funcionarios que educam a população atendida".

A partir destas claridades e para a elaboração deste texto, se tomou como referência a Fundação URDIMBRE, evidenciados pelos elementos nos momento de realizar procesos de comparação, aproximação e distanciamento dos perfis, características e competências dos e das educadoras sociais, propósitos e treinamentos dinámicos formativos que suporta a pedagogía social para a educação e qualificação docente, nos procesos sócio educativos.
\end{abstract}

Como resultado deste proceso, se pode sustentar, que a Cidade de San Juan de Pasto, tem a Fundação URDIMBRE, que por suas características e particularidades sócio educativas, pode ser localizada de forma prática mas não teórica na Educação Social, uma vez que o processo de pesquisa e reflexão, revelou que a entidade, avança nos processos de qualificação docente e investigação social, relacionada com os princípios e propósitos da Pedagogía Social, destinados à optimização da atenção e educação de diferentes grupos sociais que se encontram em situação de vulnerabilidade, deslocamento e na rua; responde as suas necessidades, tempos, interesses e expectativas, a partir de ações e metodologias que permitem a formação integral, atenção psicossocial familiar e trabalho em diferentes cénarios para a integração dos atores que fazem parte do mundo dos meninos, meninas e jovens que assistem à fundação.

Palavras chaves: Fundação URDIMBRE, perfil, competências e qualificação dos professores, Pedagogía Social. 


\section{INTRODUCCIÓN}

Niño Pavajeau (1999) sustenta que el año de 1946 fue determinante para el territorio colombiano, porque comenzó a evidenciar la redistribución de la población rural y urbana, fue definiendo sus regiones por municipios y ciudades y se presenciaron las primeras manifestaciones de desplazamiento interno debido a factores económicos, políticos y sociales y, más adelante, a situaciones como el conflicto armado en el campo y en la ciudad, dominio de tierras y, en mínimos casos, búsqueda de mejores condiciones de vida, aspectos que posteriormente influirían en el incremento de los grupos sociales vulnerables urbanos.

El Departamento de Nariño no estuvo ajeno a estos procesos y condiciones de vida; Prada Pardo, en el informe presentado por la Consultoría para los Derechos Humanos y el desplazamiento forzado (2006), sostiene que en el Departamento de Nariño, a partir de los años ochenta, surgieron las primeras avanzadas de las FARC, el ELN y M-19 en diferentes municipios, y en el 2000 cobran presencia las AUC, lo que agudiza el flagelo del conflicto y fuerza a multitud de campesinos a buscar otras posibilidades de vida y de seguridad en la ciudad de Pasto.

Esta situación de desplazamiento y asentamiento se percibió en algunas Comunas de la ciudad de Pasto, las que se reconocieron y denominaron "receptoras", al caracterizarse por la agudización de la situación de vulnerabilidad, de reubicación de población en situación de desplazamiento y de recrudecimiento de las mínimas condiciones de vida en el sentido de servicios públicos, calidad de vida y seguridad social.

Estos aspectos, directa e indirectamente, afectan y trastocan los roles y actividades de las familias receptoras; tal es el caso de algunas niñas, niños y jóvenes que hacen parte de estas Comunas, y de los otros que, en su condición de desplazamiento, llegan a estas zonas y a su corta edad asumen funciones, como el cuidado de sus hermanos menores, manutención de sus hogares y explotación laboral por parte de padres y/o familiares, entre otras situaciones.

Estas realidades develan la existencia de población de niñas, niños y jóvenes en condición de vulnerabilidad, desplazamiento y en calle, en edades comprendidas entre los cinco a los dieciocho años aproximadamente y en extra-edad escolar, características y visibilidades que no les pasaron desapercibidas a personas organizaciones sociales y educativas, de corte mixto, privado y sin ánimo de lucro, tipologías consideradas por Pérez Serrano (2003) como antecedentes para el surgimiento de la Pedagogía Social, que se debió a la "intervención subsidiaria 
del Estado por la educación de la juventud, y da lugar a diversas instituciones de iniciativa pública o privada" (44).

En estas realidades, se ubica la Fundación URDIMBRE, que en 1995 abre sus puertas para atender de manera socio-educativa a diferentes grupos sociales, que padecían circunstancias criticas generadas por factores externos e internos y que, a su vez, no los habían atendido las instituciones educativas del Estado, y otras entidades constituidas para tal finalidad.

Dicho interés lo ejecutó y llevó a cabo el señor Arturo Muñoz y la señora Liliana Bolaños, pareja de Licenciados que no veían una respuesta positiva por parte de las Instituciones Educativas Municipales a la atención de las niñas, los niños y jóvenes en situación de calle, que se encontraban en condiciones de abandono, mendicidad, maltrato familiar, en algunos casos ingerían sustancias sicoactivas y realizaban trabajos que no corresponden a su edad.

En su proceso de institucionalización y sostenibilidad, hasta el presente, la fundación ha crecido, avanzado y replanteado sus finalidades educativas, metodologías formativas - de cualificación e investigación, procesos de atención y cuidado, y apoyo sicosocial a familias, cuyos componentes, según la fundación, se ubicaron en tres frentes (niños, educadores, familias), para la mayor comprensión de la interacción al interior de las dinámicas dimensionadas y los propósitos que se procuraba alcanzar, al dejar de percibir que sus aportes van en procura de la "dinamización o activación de las condiciones educativas de la cultura, y de la vida social y sus individuos; y, por otro lado, prevención, compensación y reconducción socioeducativa de la dificultad, la exclusión o el conflicto social" (Ortega Esteban, 1999, 4).

Estos progresos y alcances de la fundación se han logrado gracias a la constitución de un equipo de trabajo cooperativo, implementación y cualificación permanente de métodos-técnicas y estrategias al interior del establecimiento, y consolidación e instauración de otras formas de educar en lo no formal y social, en escenarios diferentes a los de la educación formal o convencional, por lo que, y en el trayecto de 17 años de ejecución y atención a grupos sociales que se encuentran en situación de vulnerabilidad, desplazamiento y en calle, la fundación ha mostrado el sentido y la importancia de la Educación y Pedagogía Social en la ciudad de Pasto, además de la exigencia de volver a la dimensión social y lograr "reinventarse como una educación social en la que los objetivos de integración y convivencia sean determinantes y en la que la «escuela»-el tipo de escuela que emerja de todo este proceso de transformación- sea una instancia más de ese continuum educativo que abarca toda la vida de los hombres en la comunidad y no es algo separado y segregado de ésta" (Ortega Esteban, 1999, 3), con 
el agregado de una educación desde y para la vida y acorde a las poblaciones a las que va dirigida.

Por lo tanto, se infiere, con Antonio Petrus (1994), que esta forma de distinguir la educación y dimensionar otras opciones de educar son posibles, siempre y cuando se conceptualice y explique a la educación en "función de factores tan diversos como el contexto social, la concepción política, las formas de culturas predominantes, la situación económica y la realidad educativa del momento" (180), para evitar interpretaciones desvirtuadas o ajenas al fin o propósito que se pretenda adelantar en función de su estudio, revisión y comprensión.

\section{FUNDACIÓN SOCIAL URDIMBRE}

URDIR: Dos acepciones. Tejer, como aquella labor paciente de preparar con delicadeza y cuidado los hilos que serán la base firme y segura de un tejido entramado en mil formas y mil colores. Conspirar, entendida como el respirar juntos. Trabajar con identidad de propósitos (Muñoz, 2000).

La experiencia aparece en la ciudad de Pasto, en 1994, bajo la iniciativa de sus fundadores, Arturo Muñoz del Castillo ${ }^{1}$ y Liliana Bolaños Suárez ${ }^{2}$, pareja de Licenciados, con gran sentido de responsabilidad y compromiso social, altruista y humano, que consideraban que el propósito social de su fundación debía ser:

trabajar en los campos de la investigación, de la educación y del desarrollo social, priorizando en sus acciones las poblaciones y comunidades en condiciones especialmente difíciles y aportar en el mejoramiento de su calidad de vida; para tal efecto podrá gestionar, negociar y administrar recursos nacionales e internacionales destinados a la financiación de planes, programas y proyectos educativos y sociales que desarrollará en forma autónoma o en cooperación con otras entidades estatales o privadas ${ }^{3}$.

Su interés, como fundación, surgió de procesos investigativos sociales que llevaron a cabo en el año de 1992, en la ciudad de San Juan de Pasto, con la finalidad de realizar observaciones "para identificar las dinámicas en las diferentes horas del día, porque surgía la preocupación de que iba en aumento la población

1. Fundador de la fundación URDIMBRE, Licenciado en Matemáticas y Física de la Universidad de Nariño, Especialista en Estudios Latinoamericanos de la Universidad de Nariño; vive en la ciudad de San Juan de Pasto, casado, 61 años, autodidacta crítico de educación y pedagogía.

2. Licenciada en Pedagogía; vive en la ciudad de San Juan de Pasto, casada, 55 años; tiene formación en artes, autodidacta en pedagogía; cofundadora, educadora y coordinadora.

3. Proyecto de adecuación de la finca Janacatú; tomado del archivo de la Fundación URDIMBRE (AFU), de la carpeta apoyo para la adecuación física de la finca "Janacatú", donde se desarrolla el componente: comunidad educativa de la fundación URDIMBRE, septiembre 2007. 
de niños que permanecían en la calle y lo que se descubrió es que muy pocos no tienen familia, y permanecen o deambulaban en la calle" (A.2.a.2.17.) ${ }^{4}$.

Este aspecto les posibilitó organizarse y comenzar los procesos de recuperación de esta población, definir estrategias lúdico-pedagógicas que llamaran la atención de las niñas, niños y jóvenes ${ }^{5}$, que se encontraban en las calles de la ciudad, en los parques y en zonas de comercio y diversión, con la intención de atraerlas/os, ser amigos y, finalmente, invitarlos a adelantar procesos de sano esparcimiento, educación, atención sanitaria y formación en la sede de la "ludoteca del Barrio La Rosa" ${ }^{\prime \prime}$, ubicada en la Comuna cinco de la ciudad de San Juan de Pasto, lo cual le permitió al equipo inicial continuar con el trabajo de calle, atender a los NNJ en la ludoteca e ir estableciendo los tiempos y actividades que se llevarían a cabo en este recinto.

Estas acciones se concretan y se legalizan el 10 de abril de 1995; la fundación inicia sus labores de manera oficial, mediante Resolución 492 del 19 de octubre de 1995 de la División Jurídica de San Juan de Pasto, NIT 814.000.287-, y como representante legal se nombra al señor Arturo Muñoz del Castillo.

Para el año 1997, la fundación logra acceder a "una finca y reconstruir esa finca donde se pudiera atender a muchos más chicos; pudimos estabilizar la oferta y atenderlos todos los días de lunes a viernes en una jornada completa y organizarnos para que en la otra jornada se hiciera un trabajo en la familia y se continuara el trabajo en la calle", (A.2.a.2.19.)

Dicho inmueble se cedió en el mandato gubernamental del alcalde Antonio Navarro Wolff, en contrato de comodato por INVIPAST0 ${ }^{7}$, ubicado en San Antonio de Aranda, a una distancia de $5 \mathrm{~km}$ del centro de Pasto, con una extensión de 8.5 hectáreas.

En la organización de la finca

Participaron los chicos, los jóvenes, a su propio nivel y esto permitió trabajar desde calmar el imaginario de ir con cauchera a matar pajaritos o de maltratar el monte, a mostrar que todo tiene una validez, como apagar

4. Los códigos que se presenten de aquí en adelante, son resultado del proceso de triangulación y análisis de información de la tesis denominada "Presencia de la Pedagogía Social en los programas socio educativos de la Obra Social El Carmen y fundación URDIMBRE del Municipio de San Juan de Pasto"; dichos códigos se organizan en el Manual de códigos, ubicado en las p. 250-257.

5. De aquí en adelante, se empleara la sigla NNJ para hacer alusión a niñas, niños y jóvenes

6. En julio de 1995, la Dirección Municipal de Seguridad Social en Salud, a través de un convenio, cedió a la Fundación una casa pequeña, antiguo Centro de Salud Mental, en el barrio La Rosa de la Comuna cinco de la ciudad, para organizar la atención de las niñas y niños (Arturo Muñoz y otros, URDIMBRE, fundación educativa y social, escuela experimental urdimbre, programa de atención y formación. San Juan de Pasto: 2000), 40.

7. Instituto Municipal de la Reforma Urbana y Vivienda de Pasto, INVIPASTO. Entidad territorial del orden municipal, Pasto. 
incendios, valorar el campo, volverse investigador de pájaros, insectos, ranas, hacer clasificaciones de la reserva de todas las clases naturales que hubieran, ayudar a limpiar, pintar y a construir, las zonas de los baños de Janacatú $^{8}$ (A.2.a.2.20.2.).

En este espacio físico y natural, y por un periodo de 17 años de existencia, el equipo administrativo y de apoyo ha elaborado y ejecutado programas de formación, recuperación, asistencia sicosocial, prevención, fortalecimiento metodológico, entre otros, destinados a la atención de NNJ, denominados "niños y jóvenes de la calle" características de habitantes con problemática de calle, contraventores y consumidores de sustancias sicoactivas, que se encuentran en situación de marginalidad, maltrato, mendicidad, pobreza, abandono y desplazamiento.

De igual manera, la fundación ha buscado el proceso de recuperación individual y social, prevención de la vinculación de niños y niñas a la dinámica de calle y disminución de permanencia en la calle de aquellos que están parcial o totalmente en ella, mediante actividades como: "educación en la calle, círculos infantiles de prevención, organización de ludotecas y organización de la comunidad educativa"10.

Estas y otras acciones, que no se enuncian en este escrito, permiten vislumbrar la organización y planificación de los mecanismos pedagógicos sociales que se encaminan a la atención y formación de los NNJ, desde la mirada de la integralidad, la socialización e interacción, tres aspectos de formación, promotores del continuo aprendizaje a lo largo de la vida y que aportan las características que orientan en la comprensión de los sustentos educativos: "Primero por los procesos que ocurren a todo lo largo de la vida, desde el nacimiento hasta la

8. Janacatú significa "el sitio de arriba"; palabra indígena que invita a construir de manera permanente, sin límites en el deseo (Muñoz y otros, URDIMBRE, fundación educativa y social, escuela experimental urdimbre, programa de atención y formación (San Juan de Pasto, 1999), 39.

9. El documento nacional denominado: Análisis y mejoramiento de las condiciones de vida de los niños, niñas y jóvenes de la calle con énfasis en los que consumen sustancias psicoactivas, 1998, sostiene dos definiciones para hablar de habitantes de la calle: la primera se refiere al grupo que pasa algún tiempo en la calle, pero que normalmente duerme o realiza otras actividades cotidianas en su respectivo hogar, es receptor del maltrato físico, verbal y sicológico, lo explotan sus padres o familiares en la mendicidad $y$, en algunos casos, en la venta de drogas. La segunda se refiere al grupo para el que la calle ha reemplazado a la familia como factor esencial del crecimiento y socialización (p. 25). La Fundación URDIMBRE ha retomado la anterior catalogación y definió tres grupos para ubicar a las niñas, niños y jóvenes de la calle: el primer grupo de niñas y niños en alto riesgo, en edades de 2 a 12 años; el segundo grupo de niñas y niños en la calle, en edades de 4 a 18 años, y el tercer grupo de niñas y niños de la calle en edades de 8 a 18 años, que viven en las calles, consumen sustancias sicoactivas y la mayor parte son adictos.

10. Estas cuatro metodologías educativas, la Fundación URDIMBRE las trabaja de manera paralela con la intención de valorar y analizar los alcances y aportes formativos y humanos que están generando en las niñas, niños y jóvenes atendidos. Además, desde sus inicios en el año 1995 hasta el presente, estas metodologías se han mantenido, puesto que los resultados alcanzados han sido positivos y, en algunos casos, permiten desarticular procesos violentos y adicción a sustancias sicoactivas. 
muerte. Segundo, estos procesos tienen lugar en contextos muy diferentes (o, como suele afirmarse, a lo ancho de la vida de las personas). Y, tercero, ellos centran la atención en los aprendizajes, en vez de limitarse a la educación." (Morales y otros, 2009, 54).

Además, en esta apuesta socioeducativa, se confirman los propósitos de la Pedagogía Social, que, según Carlos Juliao (2007), procuran:

La correcta socialización de los individuos (en el marco de una formación integral cuyo referente es la persona en su globalidad, como un todo) y la intervención pedagógica para subsanar ciertas necesidades humanas que aquejan a la sociedad (lo que se llama situaciones de conflicto social). Ello, realizado normal, aunque no exclusivamente, en contextos o por medios educativos no formales (60).

Por consiguiente, queda demostrado que el trabajo socio-educativo "necesita de un cierto tiempo para ver si funciona o no, en qué medida es coherente con el discurso social del colectivo al que va orientado y si responde, o no, a las inquietudes e intereses de la población" (Añaños, 2006, 163), en lo que URDIMBRE ha sido coherente, puesto que se ha tomado su tiempo para sustentar los avances, dificultades, obstáculos y demás factores que favorecen, o no, este tipo de acciones.

\section{PERFIL, CALIDADES Y CUALIDADES DE LAS “EDUCADORAS Y EDUCADORES" ${ }^{\prime 11}$ DE LA FUNDACIÓN URDIMBRE}

Es un profesional del quehacer educativo, con un nuevo tipo de formación; no es el adulto con autoridad y poseedor de la verdad. Es el adulto que integra a las niñas, niños, jóvenes, familias y a las condiciones en que viven.

Arturo Muñoz (2000)

El equipo de trabajo, de apoyo y de formación, es importante dentro de una institución que presta servicios de atención y asistencia sicológica y de procesos formativos, porque tienen bajo su responsabilidad, humana y profesional, la interacción, el acompañamiento y el trabajo, desde varios frentes, con personas o poblaciones sociales que han presentado situaciones complejas en aspectos emocionales, sicosociales, familiares u otros.

11. Se emplea educadoras y educadores porque su fundador sustenta que "nosotros dijimos que educadores había que recuperarlo y empezamos a manejar el término; entonces, pensamos que educador era muy semejante a facilitador; ahora, "de calle" fue un apelativo que nos fuimos ganando por el tipo de trabajo; después encontramos que educador de calle lo han utilizado algunos autores y hablaban de los que trabajaban en este tipo de problemáticas; queríamos que nuestros educadores trabajaran con niños de calle". Entrevista a Arturo Muñoz, San Juan de Pasto, 25 de agosto de 2012. 
Estos desempeños se identifican y exigen, en la Educación Social, porque se requiere el desenvolvimiento del/a educador/a social en ambientes y medios específicos, tendientes a realizar y orientar acciones con individuos que presentan

Dificultades de adaptación, que requieren un soporte particular -por los motivos que sean- para integrarse a la red social. Tal tarea abarca diversas categorías de edad, desde menores a tercera edad, y diversas problemáticas: desamparo social, toxicomanías, dificultades del sujeto para circular socialmente de una manera normalizada, cuestiones en torno a la trasgresión de la ley, etc (Vélez de la Calle, 2009, 11)

Estas características del educador social, para el caso de Colombia, se las puede ubicar dentro del papel del docente, profesor/a, facilitador/a, tutor/a, $u$ otro, en la educación no formal y en propuestas educativas flexibles. En algunos casos, se los ve o considera como "agentes cuyo rol educativo está institucional o socialmente reconocido, y que no forman parte del sistema educativo graduado o que, formando parte de él, no constituyen formas estricta y convencionalmente escolares" (Gutiérrez Nieto, 2010, 2).

En criterio de Claudia Vélez de la Calle (2010), además de estas cualidades y calidades, también se debe pensar en otros requerimientos pedagógicos y sociales, como la pedagogía crítica, alternativas educativas flexibles y educación para el desarrollo, referentes para propiciar procesos analíticos y reflexivos en los educadores sociales colombianos, a fin de afrontar

Las problemáticas propias del país, producto de la inequidad, la violencia, el conflicto, y reflejada en el uso inadecuado de drogas, los desplazamientos forzados, o migraciones obligadas, los procesos de desmovilización, la reconstrucción democrática del país, la calidad de vida que deben tener los habitantes de la calle, entre otras circunstancias, propias de un desarrollo desigual que afectan a las poblaciones menos favorecidas en Colombia (11).

Dichas particularidades y condiciones entregan pautas para comprender y ubicar las finalidades y criterios de selección de personal y constitución del equipo de trabajo de la Fundación URDIMBRE, al considerar que la o el

Profesional o no profesional, formado académicamente o por autoformación, tenga disposición para formarse en URDIMBRE. Y lo escogían los niños. Si el profesional se relaciona bien con los niños, podía estar en URDIMBRE; si hay empatía y disponibilidad para trabajar esa dinámica, entonces lo contratábamos; lo fundamental es que aprenda la vida de los niños y sus dinámicas. Si no ha tenido la experiencia en eso, que tenga la disponibilidad para hacerlo (D.5.d.5.21.2). 
Por lo tanto, antes de iniciar un proceso formativo, académico y de atención, lo que prima es el reconocimiento de NNJ con quienes se interactúa: a partir de sus realidades, necesidades e intereses, se definen los procesos a ejecutar, en relación con los tiempos y dinámicas generadas al interior del grupo constituido entre la/el responsable y los individuos a atender. Por ende, una de las funciones del/a educador/a es "objetivizar el conocimiento de la situación del niño para entenderlo, poder explicar su situación. Subjetivizar es ir a lo no "medible", es ir a reconocer y valorar su opinión. Es aceptarlo tal como es." (Muñoz y otros, 2000,5)

Con estas premisas, se selecciona y contrata personal profesional y autodidacta de diferentes disciplinas, que manifiesten empatía por los principios y funciones de la fundación. En palabras de Arturo Muñoz, "que tengan disponibilidad para el trabajo, entendido éste como un proceso de construcción y cambio permanente, la aceptación de los y por los niños e interés de seguir formándose con nosotros" (D.5.d.5.21.2.).

En estas interacciones y constituciones de unidades de trabajo, se logra reconocer, de manera explícita, que las jerarquías, cargos de mando, relaciones verticales, no son los adecuados para un proceso integrador. En consecuencia, sobresale el sentido humano, social, de compromiso y de encuentro que el "equipo inicial”12 tenía como meta. En la actualidad, el sentimiento se mantiene vivo en cada uno de los miembros que hacen parte de la fundación. Esto fue posible y sostenible porque "todos trabajamos por igual, los aportes de todos importan; lo que aprendía en la universidad lo iba aplicando en Urdimbre; además, hay mucha libertad y hemos aprendido a hacer de todo, porque somos un equipo." (D.5.d.5.18).

En este sentido, es posible aceptar que las educadoras y los educadores de la fundación debían contar con unas calidades y cualidades humanas que aporten a los procesos de formación, atención y asistencia sicológica, porque tienen bajo su responsabilidad, humana y profesional, la interacción, el acompañamiento y cuidado de NNJ que han solventado diferentes experiencias que han afectado sus vidas. Conjuntamente, tienen la responsabilidad y compromiso de trabajo en varios frentes con personas o grupos sociales que han vivenciado situaciones diversas en aspectos emocionales, sicosociales, familiares u otros.

12. Según Liliana Bolaños Suárez, en entrevista realizada el 11 de julio de 2012 , "el equipo inicial fue de tres educadores de calle; yo entré como voluntaria; Arturo, que dirigía la parte pedagógica, y estaba Diego González; él tenía la parte administrativa". 
Con los anteriores sustentos, la Fundación URDIMBRE muestra, en sus referentes teóricos y vivenciales, que es posible pensar y visualizar al docente, maestro, educador, como el ser profesional que es

Investigador, reflexivo, crítico, comprometido con la situación escolar, extraescolar y sociopolítica; trabaja en equipo y transforma el contexto comunitario, escolar y el entorno social. Asume el paradigma ecológico de la pedagogía. Éste se extiende al currículo, como dentro del "mundo de la vida", en la cual se incorporan múltiples factores que tienen que ver con el complejo problema del proceso educativo (Peleteiro Vázquez, 2005, 10).

En los tiempos actuales, esto es indispensable, tanto a nivel de la educación formal, como en los programas y modelos flexibles que se están ejecutando para la atención de población que se encuentra en diversidad de situaciones y que merece una atención educativa, sicosocial, que responda y vaya acorde a sus necesidades y posibilidades.

Estos horizontes de desempeño y dedicación permiten identificar contextos en los cuales "el educador puede hacer mucho, pero no con su diagnosis, sus razonamientos, sus soluciones. El verdadero cambio lo provoca la relación de ayuda con estilo democrático, la presencia activa y vital de personalidades sólidas y que aman." (Cian, 1987, 121), caracterizaciones palpables en URDIMBRE: en sus labores se reconocen y catalogan como educadoras/es de calle; por su dedicación, entrega, compromiso profesional y, primordialmente, por su misión humanitaria y de servicio, con y para el otro.

\section{PROCESOS DE CUALIFICACIÓN Y AUTÓNOMOS VISIBLE EN LOS EDUCADORES Y EDUCADORAS DE LA FUNDACIÓN URDIMBRE}

Quien trabaja en el campo de la educación en la calle sabe una porción de cosas que descubrió, que la calle le enseñó.

Paulo Freire (1991)

Isabel Peleteiro Vásquez (2005) sustenta la necesidad de diseñar un currículo de "formación docente orientado a comprender la realidad de los sectores poblacionales en situación de exclusión y el diseño de proyectos de desarrollo social orientados a lo compensatorio y lo intercultural" (12).

Esta reflexión, y propuesta educativa y formativa para docentes, educadores y profesionales de la educación para América Latina, muestra los cambios y avances que debe enfrentar el/la educador/a del siglo XXI, porque se considera perentorio el interactuar con los diferentes grupos poblacionales con los que 
interactúa y, en algunos casos, atiende. Por ende, no es suficiente contar con una gama de teorías disciplinares y pedagógicas si no se reconoce el contexto, las condiciones, expectativas, tiempos, entre otros factores, que presentan las comunidades sociales y, sobre todo, los tipos sociales que viven en el margen del desconocimiento e invisibilización en atención y cuidado por parte del Estado.

Estos aspectos también los abordan y contextualizan en el territorio colombiano Aparicio Franco y otros, (2008), quienes sostienen que los docentes, en el siglo XXI, deben contar con las suficientes competencias, habilidades, aptitudes $\mathrm{y}$ actitudes, entre otras, para afrontar las eventualidades que se suscitan en el entorno educativo y, además, tener en cuenta el desarrollo disciplinar y profesional de las y los docentes, porque estas características posibilitan y viabilizan las

relaciones interdisciplinarias que, además del Trabajo en Equipo, exigen amplia formación humanística y una sensibilidad que deben ser ejercitadas en la acción pedagógica, la cual, está multi-determinada, puesto que participan variados actores en un ámbito compuesto de contradicciones y complejidades (3).

Estas características y condiciones posibilitan la revisión, entendimiento y observación del tipo de educación y cualificación que se está llevando a cabo por entidades públicas y privadas, en cuanto a formación de los/as futuros/as maestros/as o docentes. Y, en este proceso, es satisfactorio encontrar que la Fundación URDIMBRE, en su tiempo de funcionamiento (1995-2012), ha trabajado y tenía claridad sobre estas solicitudes, porque, desde su inicio, se fundamentó en el resultado alcanzado en las mesas de trabajo por parte del MEN en $1994 \mathrm{y}$ 1995, donde se determina que se avance en "la formación de nuevos educadores y en el desarrollo de procesos continuos de su perfeccionamiento y profesionalización, sin lo cual es muy difícil lograr procesos educativos de significación" (Muñoz y otros, 2000, 34).

Con dicho enfoque, el coordinador y su equipo de trabajo constituyeron una ruta de cualificación permanente, que contenía actividades, metodologías, estrategias didácticas y pedagógicas, "convivencias, salidas pedagógicas y cenas"13, entre otras, que promovieran el reconocimiento y trabajo colaborativo, la organización y definición de acciones que se llevarían a cabo con los individuos atendidos, el manejo de espacios y ambientes de interacción en los cuales se encontraban y avanzaban en los procesos de recuperación y formación.

13. Entrevista a Natalia Muñoz, San Juan de Pasto, 25 de junio de 2012; Psicóloga integrante del equipo de trabajo de la fundación URDIMBRE, que comenta que se hicieron muchos talleres pedagógicos y de convivencias, salidas, cenas, para conocernos y saber quiénes éramos, buscar mejores canales de comunicación y alcanzar un proceso de formación personal y grupal. 
De igual manera, se elaboraron rutas para el manejo de emociones y sentimientos por parte de los integrantes del equipo de educadores/as, trabajo con familias y líderes comunitarios, procesos de sensibilización y manejo de situaciones de violencia y conflicto, referentes de autores y escuelas para identificar y trabajar los procesos formativos por edades y necesidades, elaboración de material didáctico y lúdico desde la metodología de URDIMBRE ${ }^{14}$.

La fundación, en varias oportunidades, recibió asesoría externa, ofrecida por Profamilia, donde hubo capacitaciones sobre la parte sexual para los jóvenes y las familias, cómo hablarles a los jóvenes y niños, historia de la educación sexual; nos invitan a charlas, por ejemplo, en la [Universidad] Mariana sobre derechos de la mujer, y hace como dos años asistí a una de Publicar, sobre Internet (D.4.d.4.18).

Todo esto fue posible por la disposición y motivación de los integrantes del equipo y por los tiempos que se estipularon para estas sesiones de cualificación y elaboración de material; Liliana Bolaños $(2012)^{15}$ se refiere a estos eventos como:

unas jornadas semanales de formación del equipo; nos reuníamos mínimo una vez por semana y, en el mes, mínimo un taller mensual para profundizar sobre lo que estábamos construyendo; trabajábamos lo de investigación, todas las metodologías investigativas cualitativas y cuantitativas por los datos, mejorar la capacidad de observación; no sólo mirar, sino ver más profundamente, buscar varias fuentes de información, registrar y hacer diarios de campo para poder hacer análisis; en el tema educativo, nos centramos en mejorar la oferta en materiales didácticos y de juegos; no eran suficiente estos materiales, sino contábamos con unas guías pedagógicas que nos permitieran optimizar su proceso de autoaprendizaje con un acompañamiento personalizado.

Para cerrar este breve recorrido por la cualificación y capacitación destinada para el equipo de trabajo de la Fundación, es perentorio destacar la labor de su fundador, porque le interesaba estar al día en las corrientes pedagógicas y educativas que se gestaban a nivel internacional y nacional; participar en escenarios regionales, nacionales e internacionales con ponencias y propuestas de innovación de conceptos que implicaran la denominación y comprensión de niños y niñas en situación de calle, en calle, la normatividad estipulada para su atención y procesos formativos, manejo de niveles de estrés y consumo de sustancias sicoactivas, aspectos de género y resiliencia, entre otros, con el fiel propósito de analizarlo y trabajarlo con el grupo.

14. Los talleres y temáticas desarrolladas por la Fundación URDIMBRE desde el año 1995 se encuentran como anexos en la tesis doctoral.

15. Entrevista a Bolaños, Liliana, San Juan de Pasto, 10 de junio de 2012. 


\section{CONCLUSIONES}

El estudio de la fundación social URDIMBRE muestra cómo profesionales de diferentes disciplinas educativas y sociales, y autodidactas, trabajan mancomunadamente y de manera silenciosa a favor de niñas, niños y jóvenes que se encuentran fuera del sistema escolar, en condición de vulnerabilidad, desplazamiento y en calle, sin opciones educativas por parte del Estado que se adecúen a sus tiempos y necesidades, aspectos importantes para tener en cuenta en la educación del siglo XXI, que exige que las educadoras y educadores ingresen al mundo de estas poblaciones con estas situaciones $y$, desde estas realidades, se repiense e investigue la finalidad, funcionalidad y propósitos de la educación convencional y social, para la atención y formación de estos grupos sociales.

La fundación URDIMBRE, en sus finalidades, espacios de aprendizaje, acciones educativas y de atención sicosocial a familias y comunidad, tanto como en los perfiles, competencias, características y cualificación docente, presenta relación directa con los propósitos, población atendida, finalidad del educador, entre otros componentes propios y definidos por la Educación y Pedagogía Social. Por lo tanto, estos hallazgos permiten sustentar que es factible la revisión y estudio teórico-práctico de las fundaciones socio- educativas, a la luz de la Pedagogía Social, para reconocer y permitir el ingreso de corrientes, escuelas y referentes de autores pedagogos sociales que se han constituido a nivel mundial y que pueden abordarse y adecuarse a los diferentes contextos locales y regionales.

El estudio de la fundación URDIMBRE, en sus componentes: calidadescualidades y perfil de las y los educadores, y su cualificación deja entrever que la ciudad de San Juan de Pasto cuenta con una experiencia educativa, social y flexible, conformada por personal cualificado y competente para la formación y atención de niñas, niños y jóvenes en situación de calle, definida desde prácticas, metodologías, recursos, espacios, tiempos, entre otros, propios y definidos teóricamente por las lógicas y finalidades de la Educación y Pedagogía Social. Por lo tanto, se considera imprescindible que la Secretaria de Educación Municipal y la Gobernación del Departamento de Nariño presten más atención, apoyo y posibiliten la participación y continuidad de este tipo de fundaciones, puesto que, en este presente, a Colombia y las zonas de frontera se las considera en emergencia social y educativa, lo cual merece una atención oportuna y eficiente en cuanto a la protección, cuidado, promoción de los Derechos Humanos y ayuda a los grupos sociales que se encuentran o hacen parte del llamado "en situación de emergencia". 


\section{REFERENCIAS BIBLIOGRÁFICAS}

Añaños, Fanny y otros. Educación social, formación, realidad y retos. Granada: Grupo editorial universitario, 2006 Aparicio Franco, Ana María; Henry Hincapié Londoño; Luz Stella Palacio Salgado y María Nelsy Rodríguez Lozano. La pedagogía social y la evaluación del contexto: en la construcción de un modelo alternativo para la cualificación de maestros. Huellas de la Pedagogía social en los proceso formativos, vol. N/A, fasc. N/A, p. N/A - N/A, (2009). Facultad de Educación, Universidad Santiago de Cali, ,2010.

Ayuste, Ana; Ramón Flecha García; Fernando López y Jordi Lleras, Jordi. Planteamientos de la pedagogía crítica. Barcelona: Graó, 2005.

Cian, Luciano. El sistema educativo de Don Bosco y las líneas maestras de su estilo. Madrid: CSS, 1987

Fondo de las Naciones Unidas para la Infancia (UNICEF). Paulo Freirey los educadores de la calle- una aproximación critica- alternativas de atención a los niños de la calle. Tercera edición. Bogotá: Gente nueva, 1991.

García Garrido, José Luis. Los fundamentos de la educación social. Madrid: EMESA, 1971.

Gutiérrez Nieto, Cándido. Evaluación de programas de educación no formal. Una propuesta etnográfica naturalista, Universidad de Cádiz, 2003, http://www.uhu.es/agora/version01/digital/numeros/04/04articulos/monografico/ pdf_4/07.PDF.

Helg, Anna. La Educación en Colombia 1958-1980. En: Álvaro Tirado Mejía (ed.). La Nueva Historia de Colombia. Bogotá: Planeta, 1989.

Juliao Vargas, Carlos. Educación social. El Minuto de Dios: una experiencia y un modelo. Bogotá: Corporación Universitaria Minuto de Dios, 2007.

Morales, Marcelo y otros. Educación no formal, aportes para la elaboración de propuestas de políticas educativas. Montevideo: UNESCO, 2009, http://es.scribd.com/doc/55947892/3/Educacion-No-Formal-Politica-educativadel-MEC-2005-\%E2\%80\%93-2009.

Muñoz, Arturo; Liliana Bolaños; Natalia Muñoz y Sandra Gómez. Documento Pedagógico de la Fundación URDIMBRE. San Juan de Pasto, 2004.

Muñoz, Arturo; Liliana Bolaños; Natalia Muñoz y Sandra Gómez. Fundación educativa y social, programa de atención y formación, escuela experimental urdimbre". Segunda versión. San Juan de Pasto: noviembre 2000.

Muñoz, Arturo; Liliana Bolaños; Natalia Muñoz y Sandra Gómez. Proyecto de Autovaloración, fortalecimiento y estándares de calidad en las instituciones de protección de niños y niñas, promovido por la Fundación Restrepo Barco, la FES, el ICBF, la UNICEF y las reflexiones y aportes surgidos a partir de la gestión de legalización de la experiencia ante la SEM. Finca Janacatú, San Juan de Pasto, 2000.

Niño Pavajeau, José Francisco. Las migraciones forzadas de población, por la violencia, en Colombia: una historia de éxodos, miedo, terror, y pobreza. En: Scripta Nova. Revista Electrónica de Geografía y Ciencias Sociales, No. 45 (33). Universidad de Barcelona, (ag. 1999), http://www.ub.edu/geocrit/sn-45-33.htm

Núñez, Violeta. El educador especializado. En: Ponencias del Congreso de Educadores Sociales. Toledo: Universidad de Murcia, 1998.

Ortega Esteban, José. Pedagogía Social especializada. Barcelona: Ariel, 1999.

Peleteiro Vásquez, Isabel Helena. Pedagogía Social y Didáctica Crítica: Consideraciones para una práctica educativa orientada a los sectores en situación de desventaja y exclusión social. En: Revista de Investigación. № 58 (2005), p.49-62, Universidad Pedagógica Experimental Libertador. Instituto Pedagógico de Caracas, e-mail: isabelpeleteiro@yahoo.com.

Pérez Serrano, Gloria. Pedagogía Social /Educación Social. Construcción científica e intervención práctica. Madrid: Narcea, 2003.

Prada Pardo, Gladys. Política pública de atención al desplazamiento forzado en el Departamento de Nariño. Informe de la consultoría para los Derechos Humanos y el desplazamiento forzado, CODHES. 2006, www.codhes.org.

Petrus, Antonio. El educador social y el perfil del educador social. En: El educador social. Murcia: Universidad de Murcia, 1994.

Vélez de la Calle, Claudia. Pedagogía social en Colombia. Literatura y experiencias educativas diversas en educaciónsociedad. Cali: Universidad San Buenaventura, 2010.

Vélez de la Calle, Claudia, eds. Educación e inclusión en Colombia: una aproximación conceptual y epistemológica desde la pedagogía social. 2009, http://docs.google.com/gview?a=v\&q=cache:ZZ5CjSbW_o0J:www.amigonianos. org/SURGAM/articulos/503/COLOMBIA/MEMORIAS/tematica_completa_claudia_velez.pdf+EDUCACION+E+INC LUSION+EN+COLOMBIA:+UNA\&hl=es\&gl=co 